\title{
A!
}

This is an electronic reprint of the original article.

This reprint may differ from the original in pagination and typographic detail.

Puska, M. J.; Nieminen, R. M.

\section{Atoms embedded in an electron gas}

Published in:

Physical Review B

DOI:

10.1103/PhysRevB.27.6121

Published: 15/05/1983

Document Version

Publisher's PDF, also known as Version of record

Please cite the original version:

Puska, M. J., \& Nieminen, R. M. (1983). Atoms embedded in an electron gas: Phase shifts and cross sections.

Physical Review B, 27(10), 6121-6128. https://doi.org/10.1103/PhysRevB.27.6121

This material is protected by copyright and other intellectual property rights, and duplication or sale of all or part of any of the repository collections is not permitted, except that material may be duplicated by you for your research use or educational purposes in electronic or print form. You must obtain permission for any other use. Electronic or print copies may not be offered, whether for sale or otherwise to anyone who is not an authorised user. 


\title{
Atoms embedded in an electron gas: Phase shifts and cross sections
}

\author{
M. J. Puska \\ Laboratory of Physics, Helsinki University of Technology, SF-02150 Espoo 15, Finland \\ R. M. Nieminen \\ Department of Physics, University of Jyväskylä, SF-40100 Jyväskylä 10, Finland
}

(Received 22 November 1982)

\begin{abstract}
The Fermi-level scattering phase shifts and the transport cross sections are reported for atoms embedded in a homogeneous electron gas. The applications of the results are discussed, using the electronic stopping power for slow ions and impurity resistivity as examples.
\end{abstract}

\section{INTRODUCTION}

We have recently reported calculations of the electronic structure of atoms embedded in a homogeneous electron gas. ${ }^{1}$ The calculations are based on the density-functional method. The main focus of Ref. 1 was the energy of the embedded atom, a quantity which forms the basis of the effective-medium ${ }^{2}$ or quasiatom ${ }^{3}$ approach to chemical binding. Other aspects of such calculations are also highly interesting. The kinetic energy shifts of electrons ejected in core-level photoemission and Auger processes from free atoms and condensed phases can be obtained. ${ }^{4}$ The local density of states can be correlated with the spectral response of the embedded atom. ${ }^{1,5}$ The response of ion cores in metals to static and dynamic electric fields, i.e., the condensed-phase core polarizability, can also be calculated by this approach. ${ }^{6}$

In this paper we report results for yet another useful property of the embedded atoms: the scattering cross section they provide for Fermi-surface electrons. This quantity is expressible in terms of the scattering phase shifts in a well-known manner. ${ }^{7}$ In turn the (transport) cross section is closely related to numerous interesting physical quantities, e.g., the electron-phonon coupling constant, ${ }^{8}$ the electronic stopping power of slow ions,, 9 and the impurity resistivity. ${ }^{7}$ Moreover, Persson and Hellsing ${ }^{11}$ have recently pointed out a relation between the cross section and the damping rate of a vibrating atom.

We calculate the phase shifts using the singleparticle wave functions arising in the densityfunctional method. These wave functions are used to calculate the ground-state density of the electron system but their use in other contexts is not formally justified in the density-functional theory. However, as we shall show in this paper, the densityfunctional wave functions provide useful phase shifts in the sense that they give at least reasonable agreement with experiment. The situation is similar to the calculations in which the excited-state properties of an electronic system are determined: For example, the calculation of the properties of atoms with core holes is justified by the accuracy of the obtained core-level binding energies or ionization potentials. ${ }^{4}$

An important theme in the theory of various atomic and electronic processes in metallic condensed matter is that of local response. This means that the properties of the embedded atom can be calculated in terms of the local unperturbed host electron density around the site of the nucleus of the atom in question. For example, Persson and Hellsing ${ }^{11}$ have shown that this scheme is sufficient to explain the systematics of the damping rate of a vibrating atom on metal surfaces. Similar obervations have been made for a variety of static and dynamic processes. $^{12}$ All this underlines the usefulness of having available the phase-shift and cross-section values for atoms embedded in a homogeneous electron gas.

\section{RESULTS}

The basic theory and numerical details in the calculations have been discussed in our earlier paper. ${ }^{1}$ We embed a nucleus of charge $Z$ into a homogeneous electron gas of density $n \equiv 3 / 4 \pi r_{s}^{3}$, where $r_{s}$ is the density parameter in atomic Bohr units. We solve the Kohn-Sham density-functional equations by self-consistent iteration. The local-density approximation $^{13}$ is invoked for exchange and correlation. In the present case, only spin-compensated systems are dealt with, although the results can be generated in a straightforward manner for magnetic systems as well.

The self-consistent solution yields the scattering phase shifts $\delta_{l}(\epsilon)$ for the conduction band as a func- 
tion of energy. A number of the physical quantities can be expressed in terms of the Fermi-level phase shifts $\delta_{l}\left(\epsilon_{F}\right)$, which obey, due to the complete screening of the nuclear charge $Z$, the Friedel sum rule

$$
\frac{2}{\pi} \sum_{l}(2 l+1) \delta_{l}\left(\epsilon_{F}\right)=Z .
$$

Also the energy derivatives of phase shifts are important, because they determine the change of density of states $\Delta D(\epsilon)$ in the conduction band:

$$
\Delta D(\epsilon)=\frac{2}{\pi} \sum_{l} \frac{\partial \delta_{l}(\epsilon)}{\partial \epsilon}(2 l+1) .
$$

$\Delta D(\epsilon)$ is responsible for the impurity-induced optical properties in metals and its Fermi-level value influences electron transport quantities such as electronic specific heat. We have reported some $\Delta D(\epsilon)$ curves in Refs. 1 and 5.

The calculated $\delta_{l}\left(\epsilon_{F}\right)$ values for $Z=1$ through $Z=18$ and $r_{s}=1.5$ through $r_{s}=5$ are given in Tables I-V. For $r_{s}=2$ the $Z$ values are extended up to 40 . Partial waves up to $l=5$ are included in the tables; in the actual calculations components up to $l=10$ were used, but the phase shifts for $l>5$ are very small. The Friedel sum values [Eq. (1)] are also shown in the tables and they agree within 0.02 electrons (except for some cases with $r_{s}=1.5$ or 2) with the nuclear charge indicating a good consistency. However, in the case of $Z=1(\mathrm{H})$ with $r_{s}=2$ and
$Z=11(\mathrm{Na})$ with $r_{s}=5$ a very shallow bound $s$ state appears in the self-consistent calculations. The spatial extent of these states is comparable with the matching radius used in the calculation to fit the near-region solution to the asymptotic one. This is reflected as a slight dependence on the matching radius in the calculated phase shifts. Therefore, the phase shifts represented for these cases in the tables are not from actual self-consistent calculations but they are obtained by interpolating between the results with $r_{s}$ values, for which this $s$ state is either not bound or is strongly bound. The omissions in Tables I-V correspond to cases where we could not find a satisfactory self-consistent solution. The reason for the lack of the self-consistency in these cases is a very pronounced resonance peak in the density of states. This peak may move across the Fermi level in successive iteractions or it may sometimes form a bound state below the bottom of the band. All this means strong fluctuations in the screening cloud and achieving convergence is difficult.

Figure 1 shows in the case $r_{s}=2$ the decomposition of screening charge to different $l$ components according to the definition

$$
Z_{l}=\frac{2}{\pi}(2 l+1) \delta_{l}\left(\epsilon_{F}\right) .
$$

$Z_{0}$ is in the whole range from $Z=1$ to $Z=40$ less than the number of bound $s$ electrons in the corresponding free atoms. $Z_{1}$ follows more closely the

\begin{tabular}{|c|c|c|c|c|c|c|c|c|}
\hline \multirow[b]{2}{*}{$Z$} & \multicolumn{6}{|c|}{$\delta_{l}\left(\epsilon_{F}\right)$} & \multirow[b]{2}{*}{$\begin{array}{l}\text { Friedel } \\
\text { sum }\end{array}$} & \multirow[b]{2}{*}{$\begin{array}{c}Q \\
\left(1 / a_{0}^{2}\right)\end{array}$} \\
\hline & $l=0$ & $l=1$ & $l=2$ & $l=3$ & $l=4$ & $l=5$ & & \\
\hline 1 & 0.8528 & 0.1495 & 0.0347 & 0.0091 & 0.0025 & 0.0008 & 0.999 & 0.310 \\
\hline 2 & 1.6902 & 0.3112 & 0.0705 & 0.0177 & 0.0049 & 0.0015 & 2.012 & 0.755 \\
\hline 3 & 2.3418 & 0.5354 & 0.1060 & 0.0251 & 0.0070 & 0.0019 & 3.016 & 0.912 \\
\hline 4 & 2.8823 & 0.8148 & 0.1330 & 0.0308 & 0.0085 & 0.0020 & 4.014 & 1.112 \\
\hline 5 & 3.3630 & 1.1293 & 0.1514 & 0.0356 & 0.0095 & 0.0020 & 5.007 & 1.417 \\
\hline 6 & 3.7903 & 1.4644 & 0.1677 & 0.0407 & 0.0106 & 0.0023 & 6.002 & 1.692 \\
\hline 7 & 4.1660 & 1.8065 & 0.1886 & 0.0468 & 0.0119 & 0.0028 & 6.999 & 1.777 \\
\hline 8 & 4.5021 & 2.1432 & 0.2196 & 0.0543 & 0.0136 & 0.0036 & 8.003 & 1.631 \\
\hline 9 & 4.8158 & 2.4642 & 0.2627 & 0.0623 & 0.0154 & 0.0043 & 9.004 & 1.346 \\
\hline 10 & 5.1240 & 2.7692 & 0.3189 & 0.0706 & 0.0174 & 0.0052 & 10.017 & 1.047 \\
\hline 11 & 5.4342 & 3.0614 & 0.3846 & 0.0780 & 0.0191 & 0.0058 & 11.028 & 0.815 \\
\hline 12 & 5.7470 & 3.3477 & 0.4557 & 0.0837 & 0.0206 & 0.0061 & 12.037 & 0.690 \\
\hline 13 & 6.0570 & 3.6334 & 0.5294 & 0.0878 & 0.0216 & 0.0061 & 13.038 & 0.697 \\
\hline 14 & 6.3565 & 3.9193 & 0.6046 & 0.0907 & 0.0222 & 0.0060 & 14.030 & 0.850 \\
\hline 15 & 6.6418 & 4.2056 & 0.6878 & 0.0947 & 0.0235 & 0.0065 & 15.052 & 1.146 \\
\hline 16 & 6.9065 & 4.4829 & 0.7794 & 0.0978 & 0.0242 & 0.0067 & 16.061 & 1.539 \\
\hline 17 & 7.1514 & 4.7457 & 0.8874 & 0.1007 & 0.0250 & 0.0070 & 17.082 & 1.975 \\
\hline
\end{tabular}

TABLE I. Phase shifts at Fermi level $\delta_{l}\left(\epsilon_{F}\right)$ and Friedel sums [Eq. (1)] for atoms embedded in an electron gas with $r_{s}=1.5$. $Q$ is proportional to the electron transport cross section at the Fermi level [Eq. (4)]. 
TABLE II. Phase shifts at Fermi level $\delta_{l}\left(\epsilon_{F}\right)$ for atoms embedded in an electron gas with $r_{s}=2$. See also the caption of Table I.

\begin{tabular}{|c|c|c|c|c|c|c|c|c|}
\hline \multirow[b]{2}{*}{$Z$} & \multirow[b]{2}{*}{$l=0$} & \multicolumn{4}{|c|}{$\delta_{l}\left(\epsilon_{F}\right)$} & \multirow[b]{2}{*}{$l=5$} & \multirow{2}{*}{$\begin{array}{l}\text { Friedel } \\
\text { sum }\end{array}$} & \multirow{2}{*}{$\begin{array}{c}Q \\
\left(1 / a_{0}^{2}\right)\end{array}$} \\
\hline & & $l=1$ & $l=2$ & $l=3$ & $l=4$ & & & \\
\hline 1 & 1.0783 & 0.1213 & 0.0210 & 0.0034 & 0.0004 & 0.0000 & 1.002 & 0.269 \\
\hline 2 & 1.9814 & 0.2791 & 0.0491 & 0.0085 & 0.0018 & 0.0004 & 2.002 & 0.427 \\
\hline 3 & 2.6636 & 0.5310 & 0.0699 & 0.0108 & 0.0024 & 0.0002 & 2.996 & 0.439 \\
\hline 4 & 3.2526 & 0.8434 & 0.0754 & 0.0121 & 0.0026 & 0.0001 & 3.991 & 0.557 \\
\hline 5 & 3.7710 & 1.1934 & 0.0733 & 0.0135 & 0.0028 & 0.0001 & 4.990 & 0.749 \\
\hline 6 & 4.1990 & 1.5678 & 0.0733 & 0.0156 & 0.0030 & 0.0002 & 5.989 & 0.874 \\
\hline 7 & 4.5485 & 1.9498 & 0.0830 & 0.0191 & 0.0035 & 0.0005 & 6.992 & 0.825 \\
\hline 8 & 4.8558 & 2.3187 & 0.1073 & 0.0240 & 0.0041 & 0.0008 & 7.997 & 0.637 \\
\hline 9 & 5.1590 & 2.6588 & 0.1468 & 0.0296 & 0.0051 & 0.0013 & 9.000 & 0.428 \\
\hline 10 & 5.4860 & 2.9754 & 0.1978 & 0.0345 & 0.0060 & 0.0015 & 10.003 & 0.267 \\
\hline 11 & 5.8398 & 3.2829 & 0.2509 & 0.0375 & 0.0065 & 0.0015 & 11.001 & 0.183 \\
\hline 12 & 6.2060 & 3.5968 & 0.2997 & 0.0391 & 0.0069 & 0.0014 & 11.998 & 0.199 \\
\hline 13 & 6.5617 & 3.9232 & 0.3418 & 0.0400 & 0.0070 & 0.0012 & 12.985 & 0.338 \\
\hline 14 & 6.8921 & 4.2619 & 0.3840 & 0.0425 & 0.0076 & 0.0014 & 13.992 & 0.580 \\
\hline 15 & 7.1907 & 4.5982 & 0.4316 & 0.0453 & 0.0079 & 0.0014 & 14.990 & 0.846 \\
\hline 16 & 7.4621 & 4.9172 & 0.4940 & 0.0481 & 0.0080 & 0.0017 & 15.986 & 1.062 \\
\hline 17 & 7.7136 & 5.2073 & 0.5835 & 0.0503 & 0.0083 & 0.0021 & 17.000 & 1.219 \\
\hline 18 & 7.9455 & 5.4585 & 0.7052 & 0.0488 & 0.0079 & 0.0023 & 18.007 & 1.364 \\
\hline 19 & 8.1526 & 5.6659 & 0.8635 & 0.0438 & 0.0073 & 0.0024 & 19.014 & 1.549 \\
\hline 20 & 8.3292 & 5.8290 & 1.0581 & 0.0363 & 0.0064 & 0.0023 & 20.018 & 1.773 \\
\hline 21 & 8.4753 & 5.9532 & 1.2821 & 0.0286 & 0.0056 & 0.0021 & 21.021 & 1.971 \\
\hline 22 & 8.5970 & 6.0488 & 1.5244 & 0.0224 & 0.0050 & 0.0019 & 22.019 & 2.044 \\
\hline 23 & 8.7028 & 6.1262 & 1.7768 & 0.0183 & 0.0047 & 0.0016 & 23.016 & 1.927 \\
\hline 24 & 8.8002 & 6.1941 & 2.0328 & 0.0167 & 0.0047 & 0.0013 & 24.013 & 1.624 \\
\hline 25 & 8.8962 & 6.2601 & 2.2852 & 0.0173 & 0.0050 & 0.0012 & 25.008 & 1.209 \\
\hline 26 & 8.9991 & 6.3329 & 2.5290 & 0.0203 & 0.0055 & 0.0011 & 26.004 & 0.788 \\
\hline 27 & 9.1193 & 6.4233 & 2.7542 & 0.0257 & 0.0062 & 0.0009 & 26.996 & 0.460 \\
\hline 28 & 9.2695 & 6.5459 & 2.9491 & 0.0338 & 0.0077 & 0.0012 & 27.993 & 0.276 \\
\hline 30 & 9.6742 & 6.9249 & 3.2330 & 0.0519 & 0.0100 & 0.0014 & 29.974 & 0.276 \\
\hline 31 & 9.9036 & 7.1721 & 3.3407 & 0.0610 & 0.0115 & 0.0018 & 30.987 & 0.405 \\
\hline 32 & 10.1287 & 7.4352 & 3.4391 & 0.0687 & 0.0123 & 0.0020 & 31.986 & 0.583 \\
\hline 33 & 10.3451 & 7.7005 & 3.5397 & 0.0756 & 0.0130 & 0.0022 & 32.987 & 0.780 \\
\hline 34 & 10.5469 & 7.9494 & 3.6438 & 0.0779 & 0.0116 & 0.0014 & 33.919 & 0.970 \\
\hline 35 & 10.7480 & 8.1889 & 3.7811 & 0.0848 & 0.0136 & 0.0029 & 34.994 & 1.163 \\
\hline 36 & 10.9327 & 8.3973 & 3.9349 & 0.0851 & 0.0135 & 0.0032 & 36.002 & 1.365 \\
\hline 37 & 11.1020 & 8.5770 & 4.1140 & 0.0820 & 0.0131 & 0.0034 & 37.008 & 1.581 \\
\hline 38 & 11.2529 & 8.7283 & 4.3175 & 0.0762 & 0.0127 & 0.0035 & 38.014 & 1.781 \\
\hline 39 & 11.3850 & 8.8542 & 4.5407 & 0.0695 & 0.0123 & 0.0034 & 39.016 & 1.903 \\
\hline 40 & 11.5011 & 8.9602 & 4.7771 & 0.0637 & 0.0122 & 0.0033 & 40.017 & 1.884 \\
\hline
\end{tabular}

free-atom curve being sometimes less and sometimes more than the number of $p$ electrons in the free atoms. The $Z_{2}$ component starts to grow after $Z \geq 10$ and it is for all atoms for $Z=1$ through $Z \stackrel{\approx}{=} 40$ larger than the number of $d$ electrons in the free atoms. Figure 2 shows the decomposition in the case $r_{s}=5$, i.e., corresponding to a low-density electron gas. $Z_{0}$ and $Z_{1}$ are now closer to the freeatom counterparts than in the case $r_{s}=2 . Z_{2}$ is very small and is not shown. It is interesting to note that $Z_{0}$ and $Z_{1}$ values for $\mathrm{Ne}$ and $\mathrm{Ar}$ are very near the free-atom values. This reflects the very inert nature of the bound levels of the inert gas atoms even in the electron gas. Stott and Zaremba ${ }^{14}$ have recently 
TABLE III. Phase shifts at Fermi level $\delta_{l}\left(\epsilon_{F}\right)$ for atoms embedded in an electron gas with $r_{s}=3$. See also the caption of Table I.

\begin{tabular}{|c|c|c|c|c|c|c|c|c|}
\hline \multirow[b]{2}{*}{$Z$} & \multicolumn{6}{|c|}{$\delta_{l}\left(\epsilon_{F}\right)$} & \multirow[b]{2}{*}{$\begin{array}{l}\text { Friedel } \\
\text { sum }\end{array}$} & \multirow[b]{2}{*}{$\begin{array}{c}Q \\
\left(1 / a_{0}^{2}\right)\end{array}$} \\
\hline & $l=0$ & $l=1$ & $l=2$ & $l=3$ & $l=4$ & $l=5$ & & \\
\hline 1 & 1.3693 & 0.0534 & 0.0073 & 0.0013 & 0.0001 & 0.0000 & 1.003 & 0.163 \\
\hline 2 & 2.3648 & 0.2168 & 0.0247 & 0.0006 & 0.0002 & 0.0000 & 2.002 & 0.135 \\
\hline 3 & 3.1277 & 0.5005 & 0.0183 & -0.0018 & 0.0001 & 0.0000 & 2.998 & 0.117 \\
\hline 4 & 3.8338 & 0.8229 & -0.0040 & -0.0037 & -0.0003 & 0.0000 & 3.981 & 0.191 \\
\hline 5 & 4.3627 & 1.2016 & -0.0211 & -0.0029 & 0.0002 & -0.0004 & 4.991 & 0.307 \\
\hline 6 & 4.7014 & 1.6266 & -0.0304 & -0.0021 & 0.0001 & -0.0004 & 5.991 & 0.346 \\
\hline 7 & 4.9350 & 2.0616 & -0.0266 & 0.0000 & 0.0001 & -0.0003 & 6.993 & 0.275 \\
\hline 8 & 5.1541 & 2.4719 & -0.0039 & 0.0037 & 0.0001 & 0.0000 & 8.007 & 0.167 \\
\hline 9 & 5.4442 & 2.8217 & 0.0355 & 0.0069 & 0.0003 & 0.0002 & 9.002 & 0.085 \\
\hline 10 & 5.8577 & 3.1384 & 0.0768 & 0.0074 & 0.0002 & 0.0001 & 10.002 & 0.034 \\
\hline 11 & 6.3520 & 3.4603 & 0.1010 & 0.0057 & 0.0001 & -0.0001 & 10.999 & 0.032 \\
\hline 12 & 6.8343 & 3.8137 & 0.1063 & 0.0044 & 0.0001 & -0.0002 & 11.992 & 0.108 \\
\hline 13 & 7.2352 & 4.2089 & 0.1023 & 0.0051 & 0.0004 & -0.0004 & 12.992 & 0.242 \\
\hline 14 & 7.5495 & 4.6252 & 0.0999 & 0.0074 & 0.0004 & -0.0004 & 13.990 & 0.348 \\
\hline 15 & 7.8130 & 5.0310 & 0.1127 & 0.0113 & 0.0003 & -0.0003 & 14.991 & 0.360 \\
\hline 17 & 8.3492 & 5.7218 & 0.2160 & 0.0164 & -0.0003 & 0.0002 & 17.003 & 0.234 \\
\hline 18 & 8.6576 & 6.0004 & 0.3101 & 0.0118 & -0.0012 & 0.0004 & 18.007 & 0.191 \\
\hline
\end{tabular}

published the $Z_{l}$ decomposition for atoms of the first two rows $(Z=1, \ldots, 10)$ in electron gas with $r_{s}=3$. Their results agree with ours.

\section{DISCUSSION}

A widely useful expression derived from the phase shifts is

$$
Q=\frac{3}{k_{F} r_{s}^{3}} \sum_{l=0}^{\infty}(l+1) \sin ^{2}\left[\delta_{l}\left(\epsilon_{F}\right)-\delta_{l+1}\left(\epsilon_{F}\right)\right]
$$

where $k_{F}$ is the Fermi wave vector. This $Q$ is the Fermi-surface value for the electron transport cross section multiplied by $n k_{F}$. For a small density of

TABLE IV. Phase shifts at Fermi level $\delta_{l}\left(\epsilon_{F}\right)$ for atoms embedded in an electron gas with $r_{s}=4$. See also the caption of Table I.

\begin{tabular}{|c|c|c|c|c|c|c|c|c|}
\hline \multirow[b]{2}{*}{$Z$} & \multicolumn{6}{|c|}{$\delta_{l}\left(\epsilon_{F}\right)$} & \multirow[b]{2}{*}{$\begin{array}{c}\text { Friedel } \\
\text { sum }\end{array}$} & \multirow[b]{2}{*}{$\begin{array}{c}Q \\
\left(1 / a_{0}^{2}\right)\end{array}$} \\
\hline & $l=0$ & $l=1$ & $l=2$ & $l=3$ & $l=4$ & $l=5$ & & \\
\hline 1 & 1.5978 & -0.0164 & 0.0030 & 0.0015 & 0.0000 & 0.0000 & 1.002 & 0.098 \\
\hline 2 & 2.5916 & 0.1683 & 0.0121 & -0.0021 & 0.0001 & 0.0000 & 2.001 & 0.047 \\
\hline 3 & 3.4902 & 0.4382 & -0.0134 & -0.0047 & 0.0005 & -0.0002 & 2.997 & 0.038 \\
\hline 4 & 4.2874 & 0.7306 & -0.0377 & -0.0044 & 0.0009 & -0.0002 & 3.989 & 0.111 \\
\hline 5 & 4.7299 & 1.1372 & -0.0553 & -0.0037 & 0.0011 & -0.0004 & 4.994 & 0.188 \\
\hline 6 & 4.9413 & 1.6006 & -0.0634 & -0.0023 & 0.0009 & -0.0004 & 5.993 & 0.199 \\
\hline 7 & 5.0632 & 2.0715 & -0.0590 & -0.0001 & 0.0007 & -0.0003 & 6.993 & 0.144 \\
\hline 8 & 5.1937 & 2.5126 & -0.0383 & 0.0027 & 0.0004 & 0.0000 & 7.998 & 0.080 \\
\hline 9 & 5.4858 & 2.8711 & 0.0024 & 0.0038 & 0.0007 & 0.0000 & 9.004 & 0.039 \\
\hline 10 & 6.0327 & 3.1744 & 0.0307 & 0.0003 & -0.0003 & 0.0000 & 10.001 & 0.008 \\
\hline 11 & 6.7111 & 3.4786 & 0.0301 & -0.0027 & -0.0002 & -0.0001 & 10.998 & 0.019 \\
\hline 12 & 7.2815 & 3.8389 & 0.0143 & -0.0037 & 0.0004 & -0.0002 & 11.997 & 0.087 \\
\hline 13 & 7.6486 & 4.2735 & -0.0084 & -0.0032 & 0.0007 & -0.0004 & 12.991 & 0.167 \\
\hline 14 & 7.5495 & 4.6252 & 0.0999 & 0.0074 & 0.0004 & -0.0004 & 13.990 & 0.196 \\
\hline 17 & 8.5732 & 5.9136 & 0.0710 & 0.0064 & -0.0010 & 0.0001 & 17.002 & 0.058 \\
\hline 18 & 9.0069 & 6.2122 & 0.1285 & 0.0008 & -0.0017 & 0.0002 & 18.003 & 0.024 \\
\hline
\end{tabular}


TABLE V. Phase shifts at Fermi level $\delta_{l}\left(\epsilon_{F}\right)$ for atoms embedded in an electron gas with $r_{s}=5$. See also the caption of Table I.

\begin{tabular}{|c|c|c|c|c|c|c|c|c|}
\hline \multirow[b]{2}{*}{$Z$} & \multicolumn{6}{|c|}{$\delta_{l}\left(\epsilon_{F}\right)$} & \multirow[b]{2}{*}{$\begin{array}{c}\text { Friedel } \\
\text { sum }\end{array}$} & \multirow[b]{2}{*}{$\begin{array}{c}Q \\
\left(1 / a_{0}^{2}\right)\end{array}$} \\
\hline & $l=0$ & $l=1$ & $l=2$ & $l=3$ & $l=4$ & $l=5$ & & \\
\hline 1 & 1.7551 & -0.0738 & 0.0048 & 0.0027 & -0.0003 & 0.0000 & 1.002 & 0.059 \\
\hline 2 & 2.7348 & 0.1332 & 0.0052 & -0.0028 & 0.0004 & 0.0001 & 2.002 & 0.019 \\
\hline 3 & 3.8081 & 0.3522 & -0.0280 & -0.0033 & 0.0011 & -0.0001 & 2.999 & 0.023 \\
\hline 4 & 4.6237 & 0.6288 & -0.0455 & -0.0018 & 0.0018 & -0.0003 & 4.000 & 0.085 \\
\hline 5 & 4.9415 & 1.0695 & -0.0619 & 0.0001 & 0.0021 & -0.0005 & 5.000 & 0.131 \\
\hline 6 & 5.0499 & 1.5561 & -0.0659 & 0.0021 & 0.0014 & -0.0007 & 5.990 & 0.133 \\
\hline 7 & 5.0978 & 2.0498 & -0.0590 & 0.0043 & 0.0008 & -0.0006 & 6.992 & 0.094 \\
\hline 8 & 5.1581 & 2.5161 & -0.0412 & 0.0065 & 0.0006 & 0.0000 & 7.990 & 0.053 \\
\hline 9 & 5.4408 & 2.8910 & -0.0016 & 0.0049 & -0.0002 & 0.0001 & 9.001 & 0.027 \\
\hline 10 & 6.1308 & 3.1755 & 0.0120 & -0.0012 & -0.0001 & 0.0001 & 10.001 & 0.002 \\
\hline 11 & 7.0283 & 3.4284 & -0.0063 & -0.0020 & 0.0003 & 0.0000 & 10.995 & 0.023 \\
\hline 12 & 7.6202 & 3.7878 & -0.0038 & -0.0038 & 0.0014 & -0.0002 & 11.999 & 0.074 \\
\hline 13 & 7.9047 & 4.2585 & -0.0525 & -0.0024 & 0.0019 & -0.0006 & 12.994 & 0.121 \\
\hline 14 & 8.0284 & 4.7577 & -0.0669 & 0.0005 & 0.0015 & -0.0009 & 13.989 & 0.125 \\
\hline 17 & 8.6060 & 5.9851 & 0.0231 & 0.0049 & -0.0008 & 0.0001 & 17.001 & 0.028 \\
\hline 18 & 9.1841 & 6.2796 & 0.0550 & -0.0023 & -0.0009 & 0.0002 & 18.001 & 0.004 \\
\hline
\end{tabular}

embedded atoms, Eq. (4) gives the impurity resistivity $\Delta \rho$ when multiplied by $\hbar c_{A} / e^{2} n Z_{h}$ $=\left(c_{A} / n Z_{h}\right)(4109 \Omega)$, where $c_{A}$ is the impurity concentration in at. $\%$ and $Z_{h}$ is the number of valence electrons per host ion. ${ }^{7}$ When multiplied by $\hbar v_{I}$, where $v_{I}$ is the velocity of an ion slowly moving through the electron gas, the expression (4) gives the

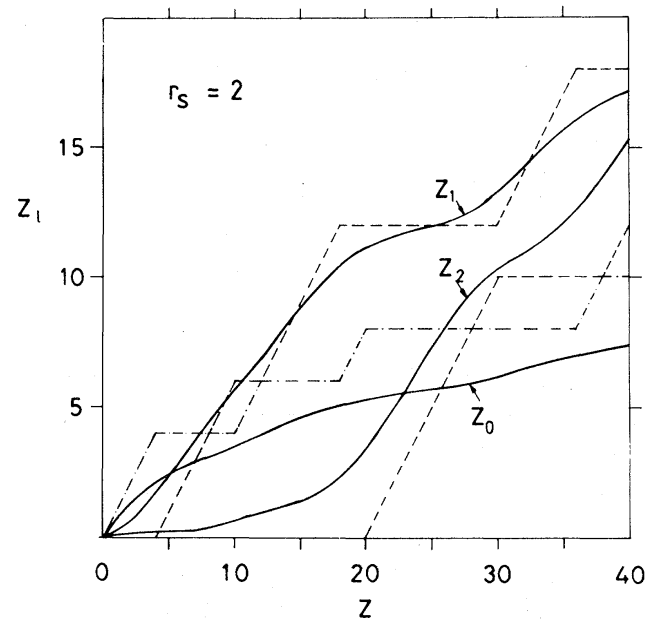

FIG. 1. Decomposition of the screening charge of atoms in electron gas to angular momentum components according to Eq. (3). The density parameter of the electron gas is $r_{s}=2$. The dash-dotted line shows the number of $s$ electrons and the dashed lines show the numbers of $p$ and $d$ electrons in free atoms, respectively. electronic contribution ${ }^{10}$ to the stopping power $d W / d r$. The damping width $\Gamma$ due to electronic excitations for a nucleus vibrating in an electron gas is obtained from Eq. (4) by multiplying by $\hbar^{2} / M$, where $M$ is the nuclear mass. ${ }^{11}$ Furthermore, the electron-phonon coupling constant $\eta$ in superconductivity theory reduces to a closely related form. ${ }^{8}$

The values of $Q$ for atoms from $\mathrm{H}$ to Ar embedded in an electron gas with $r_{s}$ parameter varying from 1.5 to 5 are listed in Tables $I-V$. Figures 3 and 4 (and also Fig. 6 for $r_{s}=2$ and $Z=1, \ldots, 40$ ) show these values as a function of $Z$ for different $r_{s}$ values. The prominent feature of the curves is the oscillations as a function of $Z$. The origin of these

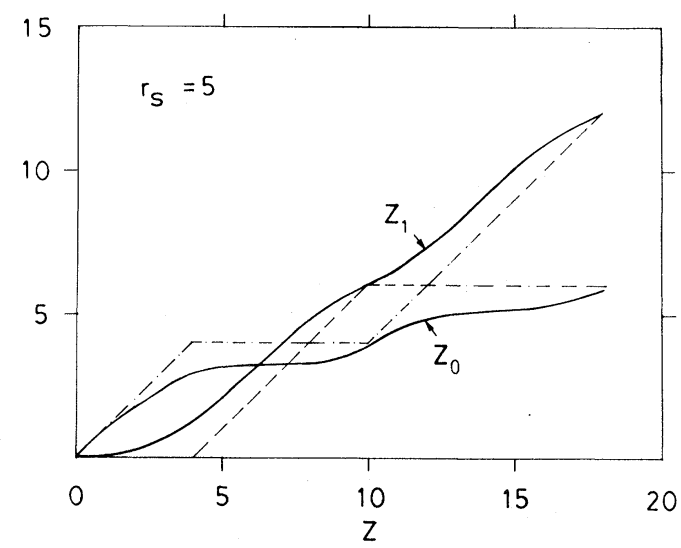

FIG. 2. Same as Fig. 1 but for $r_{s}=5$. 


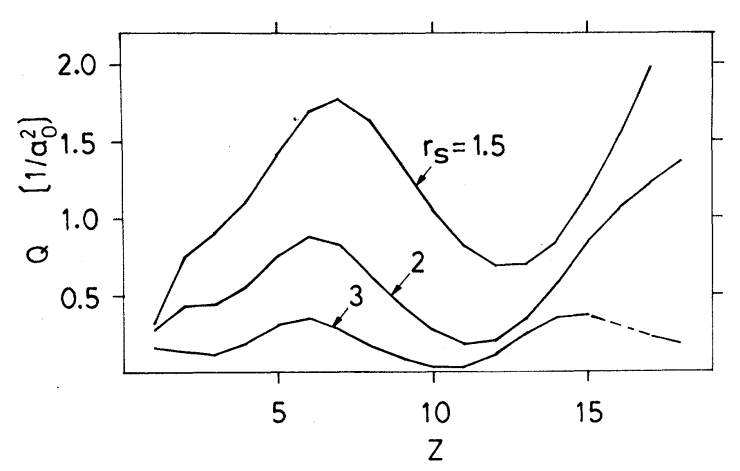

FIG. 3. Electron transport cross section at Fermi level defined via Eq. (4) for atoms in an electron gas with the density parameter $r_{s}=1.5,2$, and 3 . For $r_{s}=2$, see also Fig. 4. The dashed part of the $r_{s}=3$ curve corresponds to atoms for which we did not find fully converged solutions.

is the filling of a resonance peak in the conduction band. The oscillations can be understood by the following simple model.

The $2 p$ level is not bound for $Z=5, \ldots, 8$, and the missing electrons fill a $p$ resonance in order to satisfy complete screening. If only the resonant $p$ phase shifts are taken into account and if they are approximated from the Friedel sum rule as

$$
\delta_{1} \cong \pi(Z-4) / 6
$$

$Q$ is given by the formula

$$
Q \cong \frac{15}{k_{F} r_{s}^{3}} \sin ^{2}\left(\pi \frac{Z-4}{6}\right) .
$$

This equation predicts a maximum for $Q$ at $Z=7$. Similar arguments predict peaks between $Z=11$ and

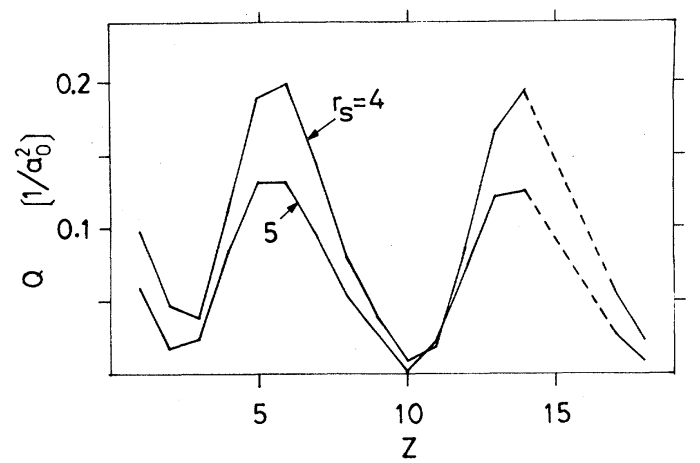

FIG. 4. Electron transport cross section at Fermi level defined via Eq. (4) for atoms in an electron gas with the density parameter $r_{s}=4$ and 5 . The dashed parts of the curves correspond to atoms for which we did not find fully converged solutions.

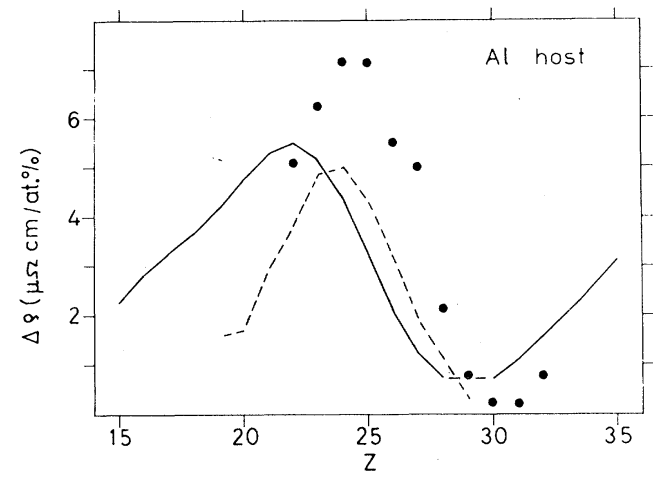

FIG. 5. Residual resistivity due to $3 d$ impurities. The solid line corresponds to a calculation, in which the phase shifts are due to atoms in an electron gas with $r_{s}=2$. The dashed line gives results obtained using the spherical solid model (Ref. 5). Experimental values (Ref. 15) denoted by dots correspond to substitutional impurities in Al.

$Z=18$, between $Z=19$ and $Z=39$, and between $Z=30$ and $Z=36$ arising from resonant $3 p, 3 d$, and $4 p$ phase shifts, respectively. When $r_{s} \leq 2$, the peaks due to the $d$-phase shifts overlap with the preceding $p$ peak and we see (Fig. 5 ) only a shoulder at $Z \approx 17$ and the $Q$ curve is smooth at $Z \approx 35$. The large contribution of the $d$-phase shifts already before the $d$ electrons become bound in free atoms is clearly seen in Fig. 1: $Z_{l}$ exceeds the number of $d$ electrons in free atoms by $\sim 3$ when $Z \approx 18$ or $Z \approx 36$. When $r_{s} \geq 3$, there are minima in $Q$ curves when $Z \approx 3$ and $Z \approx 18$. These minima appear because the screening clouds approach the free-atom electron structures when the density of the electron gas decreases (see Fig. 2). Therefore, the domination of the resonant phase shift in Eq. (4) becomes clear and limited in the $Z$ regions where the conduction-band resonance in question is filling up. This, in turn, justifies the use of the model of Eqs. (5) and (6), which predicts clearly separated oscillations.

The oscillations are seen experimentally in the impurity residual resistivity measurements and in the stopping power data for well-channeled slow ions. In Fig. 5 the experimental residual resistivity values ${ }^{15}$ due to the $3 d$ transition-metal impurities in $\mathrm{Al}$ are compared with those obtained from Eq. (4) with the phase shifts corresponding $r_{s}=2$. In the experiment the impurities are substitutional whereas our phase shifts would describe interstitial impurities. The main effect of this difference is to shift the theoretical values toward smaller $Z$ by the aluminum valence 3 . When this shift is taken into account, the calculated values reproduce the experimental behavior. The experimental values are somewhat larger, which can at least partly be explained by the lattice distortion effects which have been ignored in the calculation. The dashed line in Fig. 5 
corresponds to values ${ }^{5}$ obtained in the spherical solid model which describes a substitutional impurity. The results of these calculations are, apart from the shift, very similar to the present ones.

Experimentally it is observed that for a fixed (metallic) target material, the stopping power for slow ions shows nonmonotonic behavior as a function of the projectile nuclear charge $Z_{1}$. The periodicity of the " $Z_{1}$ oscillations" is nearly independent of target material. These oscillations were observed already about two decades ago, but their origin has been somewhat unclear until recently. A comparison of the experimental ${ }^{16,17}$ stopping-power values $\left(1 / v_{I}\right)(d W / d r)$ with the theoretical ones is given in Fig. 6. The target material in the experiment is carbon and the velocity of the ions is $v_{I}=9 \times 10^{7} \mathrm{~cm} / \mathrm{s}$ which is equal to $0.43 v_{F}\left(r_{s}=2\right)$. The experimental results with carbon target in Fig. 6 are scaled by a constant factor to give a good overall fit with theory, because the relevant atomic and electronic densities for amorphous carbon are not uniquely defined. The theoretical values are obtained from the phase shifts corresponding to $r_{s}=2$. The experimental and theoretical curves have clearly the same structure: The minima at $Z \approx 11$ and 30 and the maxima at $Z \approx 7,20$, and 38 are reproduced by the theory. One interesting detail is the "plateau" between $\mathrm{He}$ and $\mathrm{Li}$; it is seen in the experiments and it is predicted by the theory, too. However, the experimental values tend to increase more rapidly as $Z$ increases. The increasing trend is very clear in the experiment with metallic targets. ${ }^{18}$ The increase can be due to several effects. The ionic radius increases with $Z$ and therefore heavier channeling ions see a larger effective electron density than the lighter ones.

As seen from Fig. 3 the decrease of the $r_{s}$ parameter causes a strong increase in $Q$ and a filling of the minima. Evidently the increase of ionic radius also makes important slowing processes other than conduction-electron damping. One important notion also is that the velocities of ions in the experiments are usually relatively large, comparable to the Fermi velocities. The use of expression (4) becomes more justified for velocities well below $v_{F}$. Also in the experiments it is noted that the oscillations show up stronger the smaller the incident ion velocity is. ${ }^{17}$

Pathak $^{19}$ has calculated the stopping power for ions with a method somewhat similar to ours. He uses phase-shift values for electrons scattered by

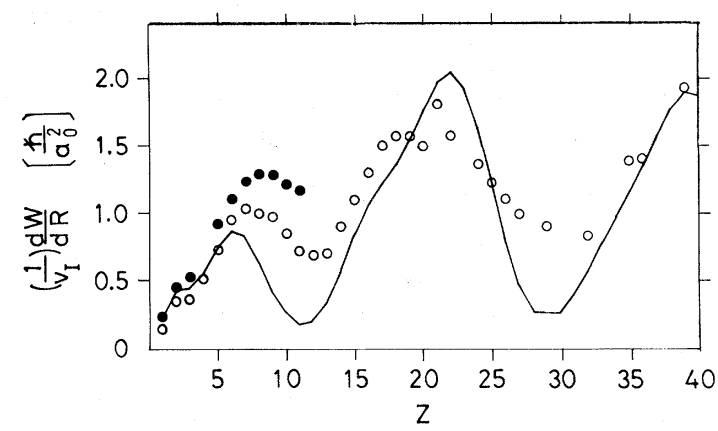

FIG. 6. Stopping power for well-channeled slow ions. Solid line gives the theoretical values obtained via Eq. (4) using phase shifts for $r_{s}=2$. Open circles and full dots denote experimental values for carbon (Refs. 16 and 17) and the aluminium (Ref. 16) targets, respectively. The experimental values with carbon target are scaled by a constant factor to give a good overall fit.

atoms, which are calculated in the Thomas-Fermi approximation, and he is able to reproduce the $Z_{1}$ oscillations. However, in his approach the phase shifts are calculated at a wave-vector value corresponding to the velocity of the incident atom. We believe that this choice of scattering wave vector is incorrect. Pathak's results are, however, similar to ours, because he uses an incident velocity very near the Fermi velocity for $r_{s}=2$.

\section{SUMMARY}

Values for the Fermi-level scattering phase shifts and the related transport cross sections have been calculated for atoms embedded in a homogeneous electron gas. These results are useful in analyzing the trends in a number of interesting physical quantities, such as the electronic stopping power for slow ions and the electron-phonon coupling constant. Within the approximation of local or quasilocal ${ }^{2,3}$ response (the density where the atom is embedded is some weighted average over the host density profile) the numbers can be directly applied to calculations which otherwise would be exceedingly difficult.

\section{ACKNOWLEDGMENT}

This work has been supported in part by the Academy of Finland. 
${ }^{1}$ M. J. Puska, R. M. Nieminen, and M. Manninen, Phys. Rev. B 24, 3037 (1981).

2J. K. Nørskov and N. D. Lang, Phys. Rev. B 21, 2131 (1980); J. K. Nørskov, ibid. 26, 2875 (1982).

${ }^{3}$ M. J. Stott and E. Zaremba, Phys. Rev. B 22, 1564 (1980).

${ }^{4}$ C. O. Almbladh and U. von Barth, Phys. Rev. B $\underline{13}$, 3307 (1976); G. W. Bryant and G. D. Mahan, ibid. $\underline{17}$, 1744 (1978); R. M. Nieminen and M. J. Puska, ibid. 25, 67 (1982).

${ }^{5}$ R. M. Nieminen and M. Puska, J. Phys. F 10 , L23 (1980).

${ }^{6}$ R. M. Nieminen and M. J. Puska, Phys. Scr. 25, 952 (1982).

${ }^{7}$ C. Kittel, Quantum Theory of Solids (Wiley, New York, 1963), p. 346.

${ }^{8}$ G. D. Gaspari and B. Gyorffy, Phys. Rev. Lett. $\underline{28}, 801$ (1972).

9J. Finneman, dissertation, The Institute of Physics, Aarhus, 1968 (unpublished).

${ }^{10}$ T. L. Ferrell and R. H. Ritchie, Phys. Rev. B 16,115 (1977).

${ }^{11}$ M. Persson and B. Hellsing, Phys. Rev. Lett. $\underline{49}, 662$ (1982).
${ }^{12}$ The local response scheme is used, for example, in the following references: M. Manninen and P. Jena, Solid State Commun. 34, 179 (1980); M. Manninen, J. K. Nørskov, and C. Umrigar, J. Phys. F 12, L7 (1982); J. K. Nørskov, Phys. Rev. Lett. 48, 1620 (1982).

${ }^{13} \mathrm{O}$. Gunnarsson and B. Lundqvist, Phys. Rev. B 13 , 4274 (1976).

${ }^{14}$ M. J. Stott and E. Zaremba, Can. J. Phys. $\underline{60}, 1145$ (1982).

${ }^{15}$ E. Babic, R. Krsnik, B. Leontic, M. Ocko, Z. Vucic, I. Zoric, and E. Girst, Solid State Commun. 10, 691 (1972); G. Boato, M. Bugo, and C. Rizzuto, Nuovo Cimento 45, 226 (1966); Y. Fukai, Phys. Rev. 186, 697 (1967).

16J. H. Ormrod and H. E. Buckworth, Can. J. Phys. 41, 1424 (1963); J. H. Ormrod, J. R. MacDonald, and H. E. Duckworth, ibid. 43,275 (1965).

${ }^{17}$ P. Hvelplund and B. Fastrup, Phys. Rev. B $\underline{165}, 408$ (1968).

${ }^{18}$ D. Ward, H. R. Andrews, I. V. Mitchell, W. N. Lennard, R. B. Walker, and N. Rud, Can. J. Phys. 57, 645 (1979).

${ }^{19}$ A. P. Pathak, J. Phys. F 4, 1883 (1974). 